\title{
Microsponge : An Overview
}

Nikita D. Gidde*, Komal A. Karande, Snehal S. Jadhav, Ruksar S. Mistry, Pratiksha

A. Mhetre, Sourabh D. Joshi

Adarsh College of Pharmacy, Vita (MS) India 415311

E-mail Id: nikitagidde19@gmail.com

\section{Address for Correspondence:}

Nikita D. Gidde

Adarsh College of Pharmacy, Vita (MS)

Email id: nikitagidde19@gmail.com

Contact Number: 9561042889 


\begin{abstract}
:
Microsponges are a revolutionary way of medication administration that has a number of advantages. The Microsponges drug delivery system is used to increase the performance of medications that are delivered orally, parenterally, or topically in a variety of conditions. Microsponge is a new technology for controlling medication release and delivering drugs to precise targets. Microsponge technology has been used in topical medicinal solutions to allow for regulated active drug release into the skin, lowering systemic exposure and reducing local cutaneous reactions to active pharmaceuticals. This review discusses the preparation procedures, evaluation methodologies, drug release mechanism, and physical characterization of Microsponges in relation to a Microsponges delivery system. Microsponges are used to deliver a pharmaceutical active component at a low dose while simultaneously improving stability, reducing adverse effects, and modifying drug release.
\end{abstract}

\title{
KEYWORDS:
}

Microsponge, topical, microsphere, drug delivery system 


\section{INTRODUCTION:}

Drug - delivery systems (DDS) that can precisely manage drug release rates or target drugs to specific body sites have a significant impact on the health-care system. By connecting the drug to a carrier particle, such as Microsponges, nanoparticles, or liposomes, which modulates the drug's release and absorption characteristics, carrier technology provides an insightful method to drug delivery. Because of their small size and effective carrier properties, microsponges are a crucial aspect of DDS. ${ }^{1,2}$

Controlling the delivery rate of active drugs to a predefined place in the human body is the biggest difficulty facing the pharmaceutical business today. Only lately has controlled medication release onto the epidermis been developed with the assurance that the drug will not enter the systemic circulation and will remain locally localised. ${ }^{1,3}$

Because of their inefficient delivery strategy, these vehicles require high concentrations of active drugs for effective therapy, resulting in discomfort and allergic responses. Other disadvantages of topical preparations include uncontrolled evaporation of the active ingredient, unpleasant odour, and the possibility of drug-vehicle incompatibility. As a result, a system that maximises the amount of time an active substance is present on the skin's surface or within the epidermis while decreasing its transdermal entry into the body is required. These requirements are met by the Microsponges delivery mechanism. ${ }^{1,4}$

\section{Microsponge $^{5,6}$}

Microsponges are porous, polymeric microspheres that are typically utilised for topical delivery over a lengthy period of time. Microsponges are used to deliver a pharmaceutically active substance at a low dose while simultaneously improving stability, reducing adverse effects, and changing drug release patterns. These characteristics have been demonstrated in the FDA-approved Retin-A Micro® $(0.1$ percent or 0.04 percent tretinoin) and Carac $(0.5$ percent 5-flurouracil) treatments for acne and actinic keratoses, respectively. ${ }^{5}$ Because of their low efficiency as delivery systems, many conventional delivery systems require significant doses of active drugs to be integrated for effective therapy. As a result, delivery mechanisms are needed to optimise the duration an active ingredient is present on the skin surface or within the epidermis while decreasing transdermal penetration into the body. Microsponge-based polymeric microspheres are the only ones that meet these requirements. Microsponges can be made in a variety of ways, including emulsion systems and suspension 
polymerization in a liquid-liquid system. Oil-in-water $(\mathrm{o} / \mathrm{w})$ is the most prevalent emulsion system, and Microsponge is made using the emulsion solvent diffusion (ESD) process.

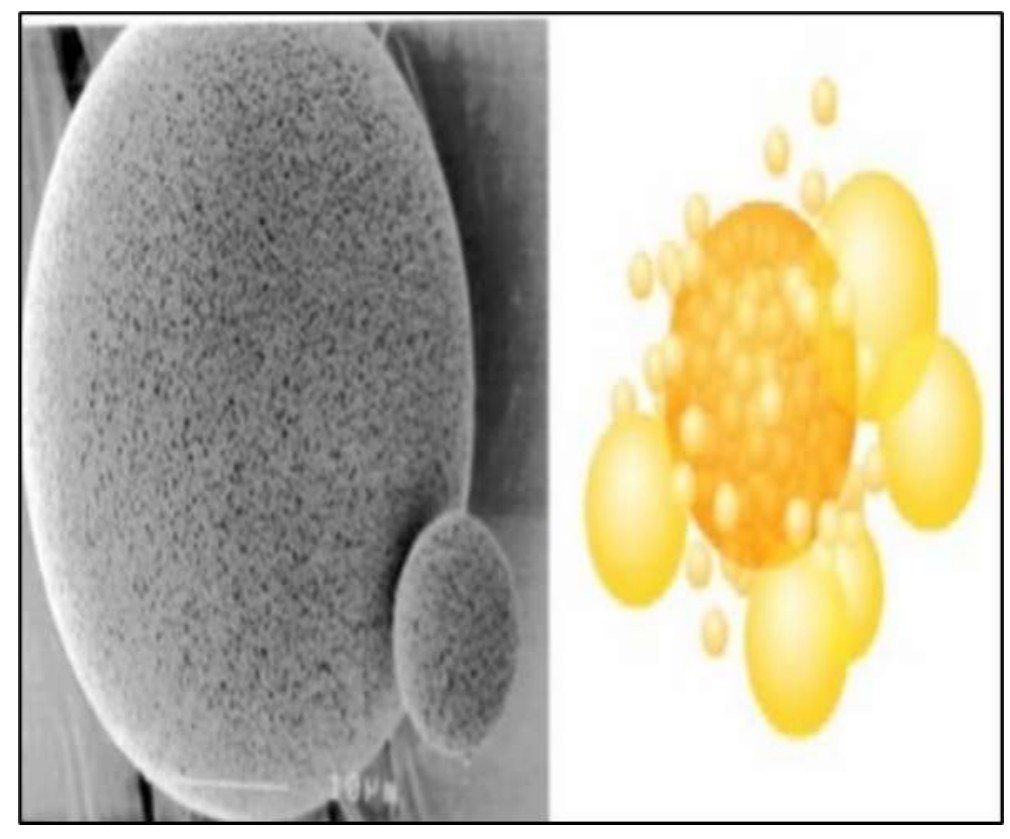

Fig 1: Microsponge Technology

\section{History of Microsponge 5}

Won invented the microsponge technique in 1987, and Advanced Polymer Systems, Inc. was given the original patents. This company created numerous variants of the technology and used them in cosmetics, as well as over-the-counter and prescription pharmaceuticals. At the moment, Cardinal Health, Inc. has been granted a licence to exploit this interesting technology in topical medications.

\section{Characteristics of microsponges: ${ }^{7,8,9}$}

1. Microsponges can withstand temperatures of up to $130^{\circ} \mathrm{C}$.

2. Microsponges are $\mathrm{pH}$-stable from 1 to 11.

3. Microsponges can be used with a wide range of substances and vehicles.

4. Microsponges are self-sterilizing because their average pore size is $0.25 \mathrm{~m}$, too small for bacteria to pass through.

5. Microsponges have a higher payload (50-60\%) while remaining free-flowing and costeffective. 


\section{Advantages of Microsponges: ${ }^{10,11,12}$}

1. The inclusion of immiscible materials is possible due to the microsponge delivery mechanism.

2. It demonstrates prolonged drug release and continued action for up to 12 hours.

3. Irritation formulae were decreased.

4. Advanced oil control; it can absorb up to six times its weight without drying.

5. Increased product opulence

6. It enables the creation of unique product forms.

7. These Microsponges are non-allergenic, non-toxic, non-mutagenic, and non-irritating.

8. Microsponges help to increase the bioavailability of the same medications.

9. It enhances condition management.

10. It improves therapeutic efficacy.

11. It improves material processing. For example, liquids can be turned into powders.

12. It improves the product's appearances, giving it a more exquisite appearance.

13. Consumer approval is high due to reduced discomfort and greater tolerance.

14. It enhances physical, chemical, and thermal stability, among other things.

\section{Characteristics of materials that is entrapped in Microsponges ${ }^{13,14}$}

1. The particles can entrap most liquid or soluble substances. The following are the conditions for actives that can be entrapped in Microsponges:

2. It must be fully miscible in monomer or capable of being made miscible by adding a small amount of a water insoluble solvent.

3. It should be water insoluble or only slightly soluble in water.

4. It should be monomer inert.

5. To minimise cosmetic issues, the solubility of actives in the vehicle must be regulated; no more than 10 to 12 percent w/w Microsponges must be included into the vehicle. Otherwise, the vehicle's Microsponges will be depleted before the application.The spherical structure of Microsponges should not collapse.

6. The Microsponges' polymer design and payload for the active must be tuned for the required release rate over a specified time period. 
7. It must be stable when in contact with the polymerization catalyst and the polymerization conditions.

\section{MECHANISM OF DRUG RELEASE ${ }^{15,16,17}$}

In reaction to one or more external triggers, microsponges can be constructed to release a specific amount of active chemicals over time.

\section{pH triggered systems}

Modifying the coating on the microsponge can be used to trigger the active's ph-based release. This has a wide range of uses in drug delivery.

\section{Temperature change}

Few entrapped active substances are too viscous to flow quickly from microsponges onto the skin at normal temperature. As the temperature of the skin rises, so does the flow rate, and hence the rate of release.

\section{Pressure}

Pressure/rubbing applied to the microsponge system can release entrapped material from the microsponges onto the skin. The amount released is determined by the sponge's many features. The microsponge most suited for a certain application can be optimised by adjusting the kind of material and other process variables. Mineral oil containing microsponge had a far greater softening effect than mineral oil containing microcapsules. The microsponge systems also have a substantially longer emollient property endurance.

\section{Solubility}

In the presence of water, microsponges containing water miscible chemicals such as antiseptics and antiperspirants will release the component. Diffusion can also be used to activate the release, however the partition coefficient of the ingredient between the microsponges and the external must be taken into account.

\section{METHOD OF PREPARATION OF MICROSPONGES ${ }^{15}$}

In general, microsponges are made using two ways: liquid-liquid suspension polymerization and quasi-emulsion solvent diffusion. However, some new procedures have recently been created, and the merits and disadvantages of these methods of production have been investigated. 


\section{Liquid -liquid suspension polymerization ${ }^{16}$}

The polymerization process continues to build a reservoir-like system with a spherical shape. The creation of chain monomers is the first step in polymerization. Ladders form as a result of cross-linking between chain monomers. Monomer ladders are folded to produce spherical particles.

The agglomeration of these micro spheres results in the formation of micro sphere bunches. Micro sponges are made by glueing together bunches of microspheres. The reaction was carried out in three necked flasks with a circular bottom. The flask is additionally equipped with a stirrer, a water condenser, and a thermometer. Monomers comprising non-polar drug solutions were produced, and an aqueous phase containing surfactant and dispersant was added to the mixture.

\section{Quasi emulsion solovent diffusion ${ }^{17}$}

The internal phase was made up of Drug, volatile Solvent, polymer, and TEC, which was added at a rate of $20 \%$ of the polymer to help with plasticity. An exterior phase containing PVA and distilled water. The internal phase was first prepared and then mixed with the external phase at room temperature. The mixture was continually swirled for 2 hours after emulsification. After that, the microsponges were separated by filtering the mixture. The product was washed and dried for 12 hours in a hot air oven at $40^{\circ} \mathrm{C}$.

\section{Water in oil in water emulsion solvent diffusion ${ }^{18}$}

In this procedure, an emulsifying agent-containing internal aqueous phase was disseminated in an organic polymeric solution. To generate a double emulsion, the water in oil emulsion was disseminated in an exterior aqueous phase containing PVA. Both water-soluble and water-insoluble medicines can be captured using this approach.

\section{Oil in oil emulsion solvent diffusion ${ }^{19}$}

The emulsion was made using this procedure because the interior phase is made up of volatile organic fluids. The volatile solvent in majority of the preparations is dichloromethane. The polymer utilised is polyactide glycolic acid, with a span of 85 as the exterior phase. To make the microsponge, the internal phase was introduced to the dispersion medium dropwise with continuous stirring.

\section{Addition of porogen ${ }^{20}$}


Porogens such as hydrogen peroxide or sodium bicarbonate make up the internal phase for this. The porogen was disseminated in the polymeric solution to form a uniform dispersion system, which was then redispersed in PVA-containing aqueous phase. When hydrogen peroxide is added, it causes the creation of linked pores with sizes ranging from 5 to $20 \mu \mathrm{m}$.

\section{Lyophilisation ${ }^{21}$}

The microspheres are transformed into porous microspheres using this process, which involves quickly removing the solvent. Chitosan hydrochloride solution is used for this. The microspheres are lyophilized after being incubated in this solution. Due to the rapid elimination of the solvent, cracking and shrinkage of microparticles may occur.

\section{Vibrating orifice aerosol generator method ${ }^{22}$}

The lipid bilayered mesoporous silica particles were largely prepared using the vibrating orifice aerosol generator method. Tetraethylorthosilicate, ethanol, water, and dilute hydrochloric acid were refluxed to prepare the stock solution for the core particle. And then this solution was diluted with a surfactant-containing solvent, resulting in monodisperse droplets. Lipsomes encase the microspheres that have been created.

These are the only microsponge preparation methods that have been described. All of the above ways have their own distinct methods of preparation; however, most microsponges are made utilising Quasi Emulsion Solvent Diffusion, which has the fewest downsides for the manufactured product when compared to other methods.

\section{CHARACTERIZATION OF MICROSPONGE ${ }^{26}$}

\section{Physicochemical properties}

\section{a) Particle size distribution:}

For particle size and size distribution, an optical microscope or an electron microscope can be employed. The texture and stability of a formulation are affected by particle size. Diffractometry or other suitable methods can be used to determine the particle size of a loaded or empty microsponge. Plotting graph particle size against time can reveal the effect of particle size on medication release.

\section{b) Determination of $\mathrm{pH}$ :}

A sophisticated $\mathrm{PH}$ metre can be used to determine the $\mathrm{pH}$ of a microsponge containing gel or another topical product. 


\section{c) Determination of true density:}

It is measured by using ultra pyanometer under helium gas.

\section{Surface Topography of Microsponges:}

For studying the surface topography of microsponges, various techniques such as photon correlation spectroscopy (PCS), SEM, and TEM can be utilised.

\section{Compatibility studies:}

TLC and FT-IR can be used to check the compatibility of the active ingredient, i.e. the medicine. Powder X-ray diffraction (XRD) and DSC are used to investigate the influence of polymerization on crystallinity.

\section{Polymer/monomer composition:}

To calculate the release rate of microsponges, a polymer composition research is required. The partition coefficient between the entrapped drug vehicle and the microsponge system is affected by polymer composition, which effects release rate. Plotting the cumulative percent of medication release against time can be used to investigate it.

\section{Viscoelastic properties:}

The viscoelastic properties of a product can be changed to meet the needs of the end product. The rate of release reduces as cross linking rises.

\section{Dissolution tests:}

Microsponge dissolution test device USP XXIII is used in conjunction with a modified basket for dissolution studies. The dissolution media is chosen based on the active ingredient's solubility. The samples were taken at appropriate intervals and analysed using appropriate analytical procedures.

\section{Kinetics of release:}

Different mathematical models were employed to analyse release data in order to research medication release mechanisms. 


\section{APPLICATIONS OF MICROSPONGES ${ }^{27}$ :}

Topical prescription, over-the-counter, and personal care products benefit from the use of a microsponge delivery method to improve their efficacy, safety, and aesthetic quality.

Microsponges have a wide range of applications. It is commonly used for topical and, more recently, oral administration. Several patents have stated that it can be employed as an excipient due to its long-term releasing ability and high loading capacity. It provides the formulator with a variety of options for developing pharmacological and cosmetic goods.

Microsponges are used to deliver a pharmaceutical active component at a low dose while simultaneously improving stability, reducing adverse effects, and modifying drug release. Numerous moisturisers, sunscreens, and specialist rejuvenation goods are available over the counter that use the microsponge medication delivery mechanism.

\section{FUTURE IMPACT OF MICROSPONGE DRUG DELIVERY SYSTEM}

Due to their unique qualities such as elegancy in appearance, performance, and pattern of release profile, microsponge drug delivery systems have a promising future in a variety of pharmaceutical applications. They also have good physical, chemical, and thermal stability, allowing for greater flexibility in dosage form manufacture. The actual future challenge will be to develop a safe medication delivery system using a variety of polymer.

\section{REFERENCES:}

1. Garish Joshi, Rajandeep Kaur, Harpreet Kaur, Microsponges: A Novel Drug Delivery System, international Research Journal of Pharmaceutical and Biosciences, 2016, 3 (1); 01- 11 .

2. Chowdary KPR and Rao YS: Mucoadhesive microspheres for controlled drug delivey. Biological Pharmaceutical Bulletin 2004; 1717-24. ,

3. Kydonieus AF and Berner B: Transdermal delivery of drugs, CRC Press, Boca Raton 1987.

4. Sandhyarani $\mathrm{R}$ and Shrinivas M: Innovative \& novel strategy: Microsphere drug delivery system. International Journal of Universal Pharmacy \& Biosciences 2014; 3: 79-93.

5. Mohite p.b.1*, khanage s.g. 1, harishchandre v.s.1, shirsath yogita1, recent advances in microsponges drug delivery system, journal of critical reviews, 2016, vol 3, issue 1, 916 
6. SK Shafi, S Duraivel, Debjit Bhowmik, KP Sampath Kumar. Microsponge drug delivery system. Indian J Res Pharm Biotechnol 2013;1:206-9.

7. Aritomi, H.; Yamasaki, Y.; Yamada, K.; Honda, H.; and Koshi, M.; Development of sustained release formulation of chlorpheniramine maleate using powder coated microsponges prepared by dry impact blending method. J. Pharm. Sci. Tech.,1996,56, 49-56.

8. Parthiban, K.G.; Manivannan, R.; Krishnarajan, D.; Chandra, S.; Nidhin Raj. Microsponge role in novel drug delivery system. Int. J. Pharma. Res. Dev.,2011, 3, 117 125.

9. Nacht, S.; Kantz, M. The Microsponge: A Novel Topical Programmable Delivery System, Topical Drug Delivery Systems. Edited by David, W.O.; and Anfon, H.A. 1992, 42, 299-325.

10. G. Ravi, valluru ravi*, p. Subhash chandra bose, damineni saritha, microsponges - a comprehensive review: success and challenges, indo american journal of pharmaceutical research, 2019, 9(07).3056-3067

11. Delattre, L.; Delneuville, I. Biopharmaceutical aspects of the formulation of dermatological vehicles. J Eur Acad Dermatol Venereol.,1995, 5, 70-71.

12. Viral Shaha et al. Microsponge drug delivery system: A review. Int. J. Res. Pharm. Sci.,2010, 1, 212-218

13. Saroj Kumar Pradhan. Microsponges as the versatile tool for drug delivery system. Int $\mathbf{J}$ Res Pharm Chem 2011;1:243-58.

14. Makwana Rajeshree, Patel Harsha, Patel Vishnu. Photostability enhancement by microsponge drug delivery system: an overview. Int J Med Pharm Res 2014;2:651-61.

15. S.A Arathy*, Sruthi Sunil, Microsponges - A New Hope for Drug Delivery System, Journal of Pharmaceutical Science \& Research 12(7), 2020, 970-972

16. Katkade Mayur, Kalkotwar Ramesh et al. Ethyl cellulose based microsponge delivery system for antifungal vaginal gel of Ticonazol, J. Drug. Deli. Thera.,2013, 14-20.

17. Christensen, M.S.; Natch, S.J. Invest. Dermato., 1983, 69, 282.

18. Sato, T., Kanke, M.; Schroeder, G.; Deluca, P. Porous biodegradable microspheres for controlled drug delivery. Assessment of processing conditions and solvent removal techniques. Pharm Res.1988, 5, 21-30.

19. Won R. Two step method for preparation of controlled release formulation. United States patent number. US5145675; 1992. 
20. Jelvehgari MR, Siahi-Shadbad S, Azarmi GP, et al. The microsponge delivery system of benzoyl peroxide: preparation, characterization and release studies. Int $\mathrm{J}$ Pharm 2006;[308]:124- 32.

21. Maiti S, Kaity S, Ray S, et al. Development and evaluation of xanthan gum-facilitated ethyl cellulose microsponges for controlled percutaneous delivery of diclofenac sodium. Acta Pharm 2011;[61]:257-70.

22. Zaki Rizkalla CM, Latif Aziz R, Soliman II. In vitro and in vivo evaluation of hydroxyzine hydrochloride microsponges for topical delivery. AAPS pharmscitech 2011;12[3]:989-1001.

23. Bae SE, Son JS, Park K, et al. Fabrication of covered porous PLGA microspheres using hydrogen peroxide for controlled drug delivery and regenerative medicine. J Control Release 2009;[133]:37-43.

24. Liu LS, Liu SQ, $\mathrm{Ng} \mathrm{SY,} \mathrm{et} \mathrm{al.} \mathrm{Controlled} \mathrm{release} \mathrm{of} \mathrm{interleukin-2} \mathrm{for} \mathrm{tumour}$ immunotherapy using alginate/chitosan porous microspheres. J Control Release 1997;[43]:65-74.

25. Lopez GP, Buranda T, Gopalaraju VRR, et al. Biologically functionalized porous microspheres. US2004005352; 2004

26. Prajakta K.Khule1*, Manoj M. Nitalikar3 , Vrunal V. More3 , Ritu M Gilhotra2, MICROSPONGE DRUG DELIVERY: A REVIEW, SGVU Journal of Pharmaceutical Research \& Education, 2019, 4(1), 359-365

27. Khopade AJ, Jain S, Jain NK. The Microsponge. East Pharm 1996;39:49-53. 